\title{
Chiral magnetic effect in condensed matter systems
}

\author{
Qiang Li \\ Condensed Matter Physics and Materials Science Department, \\ Brookhaven National Lab, Upton, New York 11973, USA

\begin{abstract}
Dmitri E. Kharzeev
Department of Physics and RIKEN-BNL Research Center, Brookhaven National Laboratory, Upton, New York 11973, USA

Department of Physics and Astronomy, Stony Brook University, New York 11794-3800, USA
\end{abstract}

\begin{abstract}
The chiral magnetic effect is the generation of electrical current induced by chirality imbalance in the presence of magnetic field. It is a macroscopic manifestation of the quantum anomaly ${ }^{1,2}$ in relativistic field theory of chiral fermions. In the quark-gluon plasma, the axial anomaly induces topological charge changing transition that results in the generation of the electrical current along the magnetic field. In condensed matter systems, the chiral magnetic effect was first predicted in the gapless semiconductors with two energy bands having pointlike degeneracies. ${ }^{3}$ Thirty years later after this prediction, the chiral magnetic effect was finally observed in the 3D Dirac/Weyl semimetals. Among the 3D Dirac semimetals is zirconium pentatelluride, $\mathrm{ZrTe}_{5}$, in which we observed a large negative magnetoresistance when magnetic field is parallel with the current. The measured quadratic field dependence of the magnetoconductance is a clear indication of the chiral magnetic effect. ${ }^{4}$ The chiral magnetic effect may emerge in a wide class of materials that are near the transition between the trivial and topological insulators. The observed phenomenon stems from the effective transmutation of Dirac semimetal into a Weyl semimetal induced by the parallel electric and magnetic fields that represent a topologically nontrivial gauge field background.
\end{abstract}


Relativistic theory of charged chiral fermions in three spatial dimensions possesses socalled chiral anomaly ${ }^{1,2}$ - non-conservation of chiral charge induced by the external gauge fields with non-trivial topology, e.g. by parallel electric and magnetic fields. Of particular interest is the chiral magnetic effect ${ }^{5}$ - the generation of electric current in an external magnetic field induced by the chirality imbalance. ${ }^{6}$ This phenomenon is currently under intense study in relativistic heavy ion collisions at Relativistic Heavy Ion Collider (RHIC) at BNL and at the Large Hadron Collider (LHC) at CERN, where it was predicted ${ }^{7}$ to induce the fluctuations in hadron charge asymmetry with respect to the reaction plane. The experimental data from the $\mathrm{STAR}^{8}$ Collaboration at RHIC and $\mathrm{ALICE}^{9}$ Collaboration at LHC indicate the fluctuations consistent with the theory expectations. Closely related phenomena are expected to play an important role in the Early Universe, possibly causing the generation of primordial magnetic fields. ${ }^{10-14}$ However, the interpretation in all these cases is under debate due to lack of control over the produced chirality imbalance.

In the field of condensed matter physics, the chiral magnetic effect is sometimes called the Adler-Bell-Jackiw anomaly. The Adler-Bell-Jackiw axis anomaly is derived from the quantum field theory and plays an important role in the standard model of particle physics. In 1983, Nielsen and Ninomiya demonstrated a similarity between the fermion system of lattice gauge theories and the electron system of crystals, and pointed out that there exists an analogous effect in condensed matter systems, for example the gapless semiconductors where two energy bands have pointlike degeneracies, and if quasi-particles behave like the Weyl fermions. This prediction remains elusive for over thirty years, because no Weyls fermions were known in any condense matter system. The recent discovery of three dimensional (3D) Dirac semimetals, $\mathrm{ZrTe}_{5}$ (Ref. 4), $\mathrm{Cd}_{3} \mathrm{As}_{2}$ and $\mathrm{Na}_{3} \mathrm{Bi}^{15-18}$ enables experimental studies of the quantum dynamics of relativistic field theory in condensed matter systems.

A prominent signature of the chiral magnetic effect in Dirac systems in parallel electric and magnetic fields is the positive contribution to the conductivity that has a quadratic dependence on magnetic field. ${ }^{5,19,20}$ This is because the chiral magnetic effect current is proportional to the product of chirality imbalance and the magnetic field, and the chirality imbalance in Dirac systems is generated dynamically through the anomaly with a rate that is proportional to the product of electric and magnetic fields. As a result, the longitudinal magnetoresistance becomes negative. ${ }^{19,20}$ 
A detailed discussion of the mechanism of the chiral magnetic effect in Dirac semimetals can be found in Ref. 4. The illustration of the chiral magnetic effect is shown in Fig. 1.
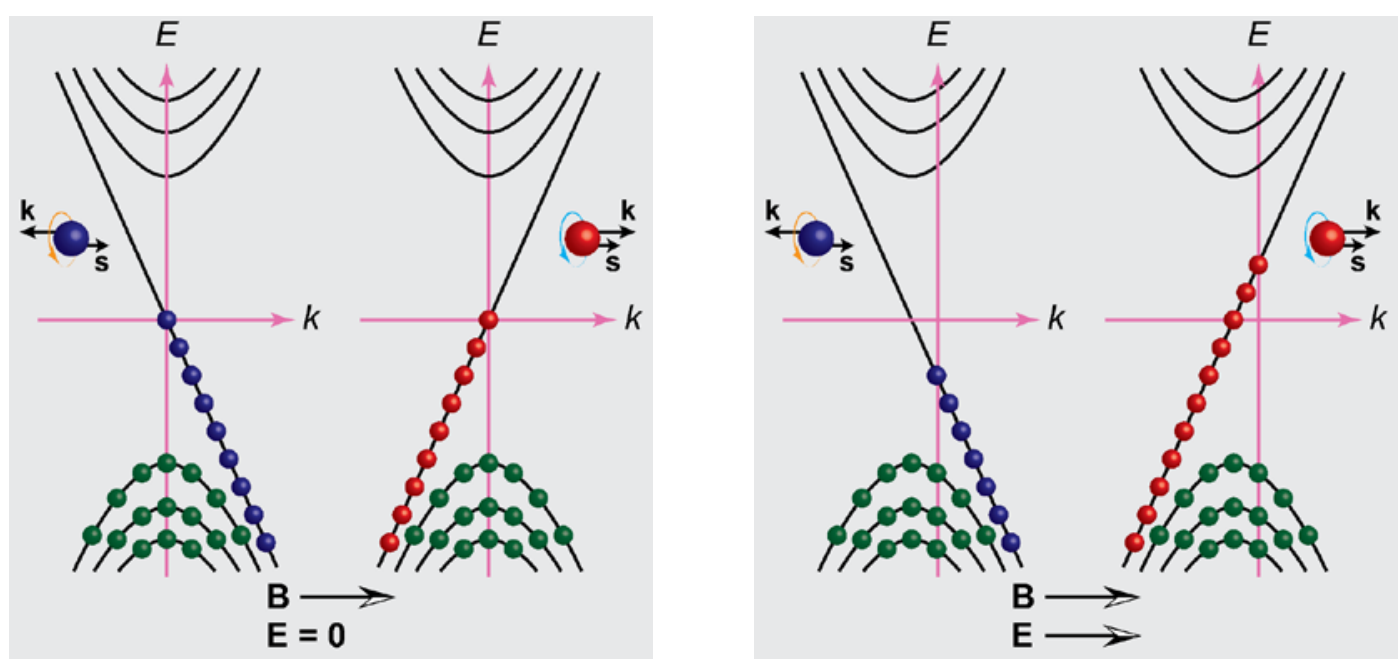

Fig. 1: Illustration of the chiral magnetic effect. Left panel: the left- and right-handed fermions occupying various Landau levels in the presence of magnetic field $\vec{B}$. On the lowest Landau level, the spins of positive (negative) chiral fermions are parallel (anti-parallel) to the external magnetic field. Therefore, for a positive fermion to be right-handed (i.e., have a positive projection of spin on momentum) means moving along the magnetic field, and for a negative fermion -- moving against the magnetic field. The left- and right-handed fermions are equally numbered under zero electric field $\vec{E}$. Right panel: Under external electric field parallel to magnetic field, the positive (negative) fermions accelerate (decelerate) along the electric field that is also parallel to magnetic field direction. This creates a non-zero chemical potential, leading to a net chiral magnetic effect current.

In the absence of external fields, each Dirac point in Dirac semimetals initially contains left- and right-handed fermions with equal chemical potentials. If the energy degeneracy between the left- and right-handed fermions gets broken, a chiral chemical potential $\mu_{5}$ is generated with the corresponding density of chiral charge given by ${ }^{6}$

$$
\rho_{5}=\frac{\mu_{5}^{3}}{3 \pi^{2} v^{2}}+\frac{\mu_{5}}{3 v^{3}}\left(T^{2}+\frac{\mu^{2}}{\pi^{2}}\right)
$$


where $\mu=\left(\mu_{R}+\mu_{L}\right) / 2$ and $T$ are the averaged chemical potential of the left and right handed fermions and the temperature, and $v$ is the Fermi velocity.

Under the external electric and magnetic fields, the generation rate of chiral charge is given by

$$
\frac{d \rho_{5}}{d t}=\frac{e^{2}}{4 \pi^{2} \hbar^{2} c} \vec{E} \cdot \vec{B} .
$$

The chirality-changing scattering can mix the left- and right-handed fermions in Dirac semimetals. By introducing the chirality-changing scattering time $\tau_{\mathrm{v}}$, we arrive at the equation

$$
\frac{d \rho_{5}}{d t}=\frac{e^{2}}{4 \pi^{2} \hbar^{2} c} \vec{E} \cdot \vec{B}-\frac{\rho_{5}}{\tau_{V}} .
$$

The solution of equation (3) at $t>>\tau_{\mathrm{V}}$ is

$$
\rho_{5}=\frac{e^{2}}{4 \pi^{2} \hbar^{2} c} \vec{E} \cdot \vec{B} \tau_{V} .
$$

According to equation (1), this leads to a non-zero chiral chemical potential $\mu_{5}$ (we assume that $\left.\mu_{5}<<\mu, T\right):$

$$
\mu_{5}=\frac{3}{4} \frac{v^{3}}{\pi^{2}} \frac{e^{2}}{\hbar^{2} c} \frac{\vec{E} \cdot \vec{B}}{T^{2}+\frac{\mu^{2}}{\pi^{2}}} \tau_{V} .
$$

As illustrated in Fig. 1, on the lowest Landau level, the spins of positive (negative) chiral fermions are parallel (anti-parallel) to the external magnetic field. Therefore, for a positive fermion to be right-handed (i.e., have a positive projection of spin on momentum) means moving along the magnetic field, and for a negative fermion -- moving against the magnetic field. The system develops a net electric current that can be computed ${ }^{6}$ by field-theoretical method and is given by

$$
\vec{J}_{\mathrm{CME}}=\frac{e^{2}}{2 \pi^{2}} \mu_{5} \vec{B} .
$$

The formulae (6) and (5) yield the final expression for the chiral magnetic effect current:

$$
J_{\mathrm{CME}}^{i}=\frac{e^{2}}{\pi \hbar} \frac{3}{8} \frac{e^{2}}{\hbar c} \frac{v^{3}}{\pi^{3}} \frac{\tau_{V}}{T^{2}+\frac{\mu^{2}}{\pi^{2}}} B^{i} B^{k} E^{k} \equiv \sigma_{\mathrm{CME}}^{i k} E^{k} .
$$


When the electric and magnetic fields are parallel, the chiral magnetic effect conductivity is

$$
\sigma_{\mathrm{CME}}^{z Z}=\frac{e^{2}}{\pi \hbar} \frac{3}{8} \frac{e^{2}}{\hbar c} \frac{v^{3}}{\pi^{3}} \frac{\tau_{V}}{T^{2}+\frac{\mu^{2}}{\pi^{2}}} B^{2} .
$$

Since the CME current is directed along the electric field, it will affect the measured conductivity as the total current will be the sum of the Ohmic and the chiral magnetic effect (CME) ones:

$$
J=J_{\mathrm{Ohm}}+J_{\mathrm{CME}}=\left(\sigma_{\mathrm{Ohm}}+\sigma_{\mathrm{CME}}\right) E,
$$

where $\sigma_{\mathrm{CME}} \equiv \sigma_{\mathrm{CME}}^{Z Z}$. If the electric and magnetic fields are parallel, there is no conventional contribution to magnetoresistance induced by the Lorentz force. The magnetoconductance (8) has a characteristic quadratic dependence on magnetic field. It is precisely this contribution to magnetoconductance with a quadratic dependence on magnetic field that we have unambiguously observed in $\mathrm{ZrTe}_{5}$ for the first time. ${ }^{4}$
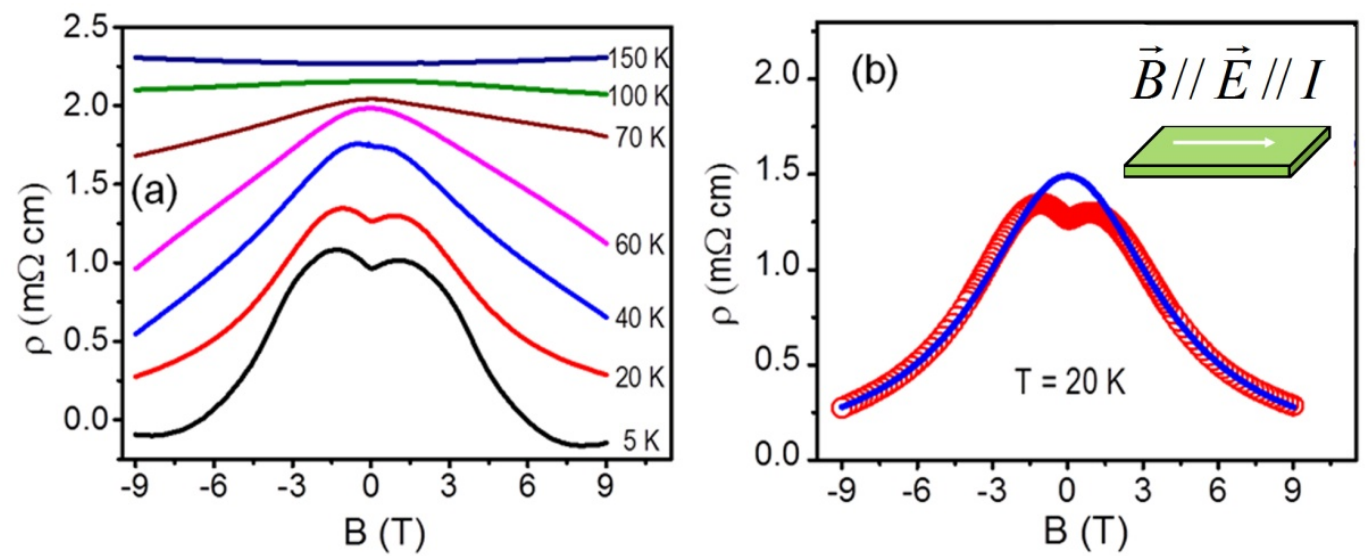

Fig. 2: Magnetoresistance in field parallel to current $(\vec{B} \| \vec{E})$ in $\operatorname{ZrTe}_{5}$. (a) MR at various temperatures. For clarity, the resistivity curves were shifted by $1.5 \mathrm{~m} \Omega \mathrm{cm}(150 \mathrm{~K}), 0.9 \mathrm{~m} \Omega \mathrm{cm}$ (100 K), $0.2 \mathrm{~m} \Omega \mathrm{cm}(70 \mathrm{~K})$ and $-0.2 \mathrm{~m} \Omega \mathrm{cm}(5 \mathrm{~K})$. (b) MR at $20 \mathrm{~K}$ (red symbols) fitted with the chiral magnetic effect curve (blue line); inset: sample geometry with $\vec{B}$ and $\vec{E}$ along the $a$-axis of the single crystal.

Fig. 2 shows the magnetoresistivity (MR) at various temperatures in a magnetic field parallel to the current in $\mathrm{ZrTe}_{5}$. At elevated temperatures, $T \geq 110 \mathrm{~K}$, the $\rho$ vs $B$ curves show a 
small upward curvature, a contribution from inevitable perpendicular field component due to an imperfect alignment between current and magnetic field. In fact, the small perpendicular field contribution to the observed resistivity can be fitted with a simple quadratic term. This term is treated as a background and subtracted from the parallel field component for all MR curves recorded at $T \leq 100 \mathrm{~K}$.

A negative MR is observed for $T \leq 100 \mathrm{~K}$, increasing in magnitude as temperature decreases. We found that the magnetic field dependence of the negative MR can be nicely fitted with the CME contribution to the electrical conductivity, given by $\sigma_{\mathrm{CME}}=\sigma_{0}+a(T) B^{2}$, where $\sigma_{0}$ represents the zero field conductivity. The fitting is illustrated in Fig. 2(b) for $T=20 \mathrm{~K}$, with an excellent agreement between the data and the CME fitting curve. At 4 Tesla, the CME conductivity is about the same as the zero-field conductivity. At 9 Tesla, the total magnetoconductivity increases by $\sim 400 \%$ over the zero field value due to the CME contribution. This results in a negative MR that is much stronger than any conventional one reported at an equivalent magnetic field in a non-magnetic material. To demonstrate that the effect is not limited to any specific crystal direction, but only locked to the current direction, we have also performed magnetoresistance measurements with the current still in the $a-c$ plane, but 18 degree off the chain direction ( $a$-axis). In this direction, we observe the same $\propto B^{2}$ dependence of CME when magnetic field is parallel to current.

At very low field, the data show a small cusp-like feature. The origin of this feature is not completely understood, but it probably indicates some form of weak anti-localization (WAL). This and the large positive magneto-resistance for magnetic field perpendicular to current, seem to be common features in all the materials that were subsequently found to display the CME in parallel fields.

After submission of our original manuscript ${ }^{21}$ to the arXive in December 2014, we noticed a surging interest in the study of magnetotransport in Dirac-like semimetals, with several recent preprints reporting a negative magneto-resistance as a consequence of the chiral magnetic effect. The effect is observed in two types of 3D chiral materials. The first type is a Dirac semimetal, where the Dirac points for left- and right-handed fermions are not separated in momentum space. These materials are effectively transformed into Weyl semimetals by parallel electric and magnetic fields, with imbalance between the densities of left- and right-handed fermions induced by the chiral anomaly. Members of that class are $\mathrm{ZrTe}_{5}, \mathrm{Na}_{3} \mathrm{Bi}^{22}, \mathrm{Cd}_{3} \mathrm{As}_{2}{ }^{27}$, 
and $\mathrm{Bi}_{1-\mathrm{x}} \mathrm{Sb}_{\mathrm{x}}(\mathrm{x} \approx 0.03)^{28}$. In particular, a very detailed transport measurement was performed recently with Dirac semimetal $\mathrm{Na}_{3} \mathrm{Bi}^{22}$, with results similar to ours. They observed both a cusp of longitudinal MR near zero magnetic field and a negative longitudinal MR in stronger fields due to the chiral magnetic effect, with a steep dependence on the angle between electric and magnetic fields ${ }^{22}$. The second type of 3D chiral materials is inversion asymmetric Weyl semimetal, like $\mathrm{TaAs}^{23}, \mathrm{NbAs}^{24}, \mathrm{NbP}^{25}$ and $\mathrm{TaP}^{26}$. In both types of materials, parallel electric and magnetic fields are required to observe the chiral magnetic effect.

\section{Acknowledgements}

The writing of this preceding paper was supported by the US Department of Energy, Office of Basic Energy Sciences, under Contract No. DE-SC0012704. The author thanks Prof. Dmitri E. Kharzeev for valuable theoretical inputs.

\section{References}

1. S. L. Adler. Axial-vector vertex in spinor electrodynamics. Physical Review, 177, 2426 (1969).

2. J. S. Bell and R. Jackiw. A PCAC puzzle: $\pi^{0} \rightarrow \gamma \gamma$ in the $\sigma-m o d e l$. Il Nuovo Cimento A, 60, 47-61 (1969).

3. H. B. Nielsen and M. Ninomiya. The Adler-Bell-Jackiw anomaly and Weyl fermions in a crystal.Physics Letters B, 130, 389-396 (1983).

4. Q. Li, D. E. Kharzeev, C. Zhang, Y. Huang, I. Pletikosić, A. V. Fedorov, R. D. Zhong, J. A. Schneeloch, G. D. Gu, and T. Valla, “Chiral magnetic effect in ZrTe, Nature Physics (2016) doi:10.1038/nphys3648.

5. K. Fukushima, D. Kharzeev, and H. Warringa. Chiral magnetic effect. Phys. Rev. D, 78, 074033 (2008).

6. D. E. Kharzeev. The chiral magnetic effect and anomaly-induced transport. Progress in Particle and Nuclear Physics, 75, 133-51 (2014). 
7. D. Kharzeev. Parity violation in hot QCD: Why it can happen, and how to look for it. Physics Letters B, 633, 260-264 (2006).

8. B. Abelev, M. Aggarwal, Z. Ahammed, A. Alakhverdyants, B. Anderson, D. Arkhipkin, G. Averichev, J. Balewski, O. Barannikova, L. Barnby, et al. Azimuthal charged-particle correlations and possible local strong parity violation. Physical review letters, 103, 251601 (2009).

9. B. Abelev, J. Adam, D. Adamova, A. Adare, M. Aggarwal, G. A. Rinella, A. Agocs, A. Agostinelli, S. A. Salazar, Z. Ahammed, et al. Charge separation relative to the reaction plane in $\mathrm{Pb}$-Pb collisions at $\sqrt{S_{N N}}=2.76 \mathrm{TeV}$. Physical review letters, 110, 012301 (2013).

10. A. Vilenkin and D. Leahy. Parity nonconservation and the origin of cosmic magnetic fields. The Astrophysical Journal, 254, 77-81 (1982).

11. J. Fröhlich and B. Pedrini. New applications of the chiral anomaly. Preprint at http://arxiv.org/pdf/hep-th/0002195.pdf

12. M. Joyce and M. Shaposhnikov. Primordial magnetic fields, right electrons, and the Abelian anomaly. Physical Review Letters, 79, 1193 (1997).

13. M. Giovannini and M. E. Shaposhnikov. Primordial hypermagnetic fields and the triangle anomaly. Physical Review D, 57, 2186 (1998).

14. T. Vachaspati. Estimate of the primordial magnetic field helicity. Physical review letters, 87, 251302 (2001).

15. Z. Wang, Y. Sun, X.-Q. Chen, C. Franchini, G. Xu, H. Weng, X. Dai, and Z. Fang. Dirac semimetal and topological phase transitions in $A_{3} B i(A=N a, K, R b)$. Phys. Rev. B, 85, 195320 (2012).

16. Z. Wang, H. Weng, Q. Wu, X. Dai, and Z. Fang. Three-dimensional Dirac semimetal and quantum transport in $\mathrm{Cd}_{3} A s_{2}$. Phys. Rev. B, 88, 125427 (2013).

17. S. Borisenko, Q. Gibson, D. Evtushinsky, V. Zabolotny, B. Büchner, and R. J. Cava. Experimental Realization of a Three-Dimensional Dirac Semimetal. Phys. Rev. Lett., 113, 027603 (2014).

18. Z. K. Liu, B. Zhou, Y. Zhang, Z. J. Wang, H. M. Weng, D. Prabhakaran, S.-K. Mo, Z. X. Shen, Z. Fang, X. Dai, Z. Hussain, and Y. L. Chen. Discovery of a three-dimensional topological Dirac semimetal, $\mathrm{Na}_{3} B$ Bi. Science (New York, N.Y.), 343, 864-7 (2014). 
19. D. T. Son and B. Z. Spivak. Chiral anomaly and classical negative magnetoresistance of Weyl metals. Physical Review B, 88, 104412 (2013).

20. A. A. Burkov. Chiral Anomaly and Diffusive Magnetotransport in Weyl Metals. Physical Review Letters, 113, 247203 (2014).

21. Q. Li, D. E. Kharzeev, C. Zhang, Y. Huang, I. Pletikosic, A. V. Fedorov, R. D. Zhong, J. A. Schneeloch, G. D. Gu, T. Valla. Observation of the chiral magnetic effect in $\mathrm{ZrTe}_{5}$. arXiv:1412.6543

22. J. Xiong, S. K. Kushwaha, T. Liang, J. W. Krizan, M. Hirschberger, W. Wang, R. J. Cava, and N. P. Ong. Evidence for the chiral anomaly in the Dirac semimetal $\mathrm{Na}_{3} \mathrm{Bi}$. Science (New York, N.Y.), 350, 413-416 (2015).

23. X. Huang, L. Zhao, Y. Long, P. Wang, D. Chen, Z. Yang, H. Liang, M. Xue, H. Weng, Z. Fang, X. Dai, and G. Chen. Observation of the Chiral-Anomaly-Induced Negative Magnetoresistance in 3D Weyl Semimetal TaAs. Physical Review X, 5, 031023 (2015).

24. X. Yang, Y. Li, Z. Wang, Y. Zhen, and Z-a. Xu. Observation of Negative Magnetoresistance and nontrivial $\pi$ Berrys phase in $3 D$ Weyl semi-metal NbAs. Preprint at http://arxiv.org/abs/1506.02283.

25. Z. Wang, Y. Zheng, Z. Shen, Y. Zhou, X. Yang, Y. Li, C. Feng, and Z.-A. Xu. Helicity protected ultrahigh mobility Weyl fermions in NbP. Preprint at http://arxiv.org/abs/1506.00924.

${ }^{26 .}$ C. Shekhar, F. Arnold, S.-C. Wu, Y. Sun, M. Schmidt, N. Kumar, A. G. Grushin, J. H. Bardarson, R. Donizeth dos Reis, M. Naumann, M. Baenitz, H. Borrmann, M. Nicklas, E. Hassinger, C. Felser, and B. Yan. Large and unsaturated negative magnetoresistance induced by the chiral anomaly in the Weyl semimetal TaP. Preprint at http://arxiv.org/abs/1512.04229.

27. C.-Z. Li, L.-X. Wang, H. Liu, J. Wang, Z.-M. Liao, and D.-P. Yu. Giant negative magnetoresistance induced by the chiral anomaly in individual $\mathrm{Cd}_{3} \mathrm{As}_{2}$ nanowires. Nat. Commun. 6:10137 doi: 10.1038/ncomms10137 (2015).

${ }^{28 .}$ H.-J. Kim, K.-S. Kim, J.-F. Wang, M. Sasaki, N. Satoh, A. Ohnishi, M. Kitaura, M. Yang, and L. Li. Dirac versus Weyl Fermions in Topological Insulators: Adler-Bell-Jackiw Anomaly in Transport Phenomena. Phys. Rev. Lett., 111, 246603 (2013). 